\title{
CEBAF celebrates seven years of physics
}

Douglas Higinbotham reports from the Jefferson Lab symposium on results that span the boundary between nuclear-meson models and quark-gluon physics.

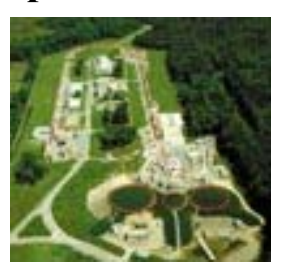

CEBAF Jefferson Lab in Newport News, Virginia, recently celebrated the first seven years of physics with the Continuous Electron Beam Accelerator Facility, CEBAF. The unique design of this electron accelerator allows three experimental halls to be operated simultaneously, with a total beam current of $200 \mu \mathrm{A}$ and a beam polarization of up to $80 \%$. With this facility, a user community of more than 1000 scientists from 187 institutions in 20 countries has completed 81 nuclear-physics experiments, with substantial data taken on 23 more. From the data obtained in these experiments, more than 250 refereed journal articles have been published and 146 doctoral degrees have been awarded. In the near future more than 60 experiments are planned, and there are currently $128 \mathrm{PhD}$ theses in progress.

To celebrate and review these accomplishments, while also looking toward the future, the Jefferson Lab user group board of directors organized a symposium, which was held on 11-13 June and dedicated to the memory of Nathan Isgur, Jefferson Lab's first chief scientist. The meeting was divided into eight physics topics: nucleon form factors, fewbody physics, reactions involving nuclei, strangeness production, structure functions, parity violation, deep exclusive reactions and hadron spectroscopy. Each topic was presented by one experimentalist and one theorist.

The symposium began with presentations by Donal Day of Virginia and John Ralston of Kansas on nucleon form factors, which probe the electromagnetic structure of the proton and neutron. The presentations included a discussion of the most referenced and surprising result from Jefferson Lab, that the proton's form factors do not follow an expected simple relation. While theorists have proposed different models to explain this result, the basic ingredient in almost all new models is the addition of relativistic effects.

The talks continued with presentations focusing on few-body systems, such as the deuteron and ${ }^{3} \mathrm{He}$, by Paul Ulmer of Old Dominion University and Franz Gross from the College of William and Mary. In these experiments, the Jefferson Lab electron beam is used to knock out a proton from the few-body system or to probe it with elastic scattering. The expected yield can be calculated exactly, assuming nucleons and mesons are the underlying particles. The presentations showed that even with beam energies of up to $5.7 \mathrm{GeV}$, the electron scattering results are surprisingly well explained by the nucleon-meson models to distance scales of the order of $0.5 \mathrm{fm}$. In contrast, experiments on deuteron photodisintegration, which probe even smaller distance scales, have revealed clear evidence of the limitations of the nucleon-meson models and of the onset of quarkgluon degrees of freedom.

For reactions involving nuclei, i.e. many-body systems such as oxygen and carbon, statistical methods in the context of the nucleon-meson picture are used to calculate the expected yields of the quasi-elastic reaction. Larry Weinstein of Old Dominion University presented a talk entitled "So where are the quarks?", in which he showed that the nucleon-meson model describes even the highest momentum transfer Jefferson Lab data, while Misak Sargsian of Florida International presented a talk looking mostly to the future, when the quark-gluon nature of matter should become evident from experiments with a $12 \mathrm{GeV}$ electron beam. 
Reinhard Schumacher of Carnegie Mellon and Steve Cotanch of North Carolina State presented reactions involving strangeness production, which includes the production of particles such as kaons. They showed new Jefferson Lab data confirming the $\theta^{+}$particle as discovered by SPring-8 in Japan (CERN Courier September 2003 p5). This new particle is comprised of five quarks and has been dubbed the pentaquark. This had been described as the first observed nucleon resonance comprised of more than three valence quarks and has sparked international excitement. A new Jefferson Lab experiment to further study this new particle has already been approved.

Structure-function experiments, which provide information on the quark and gluon structure of the nucleon, were presented by Keith Griffioen of the College of William and Mary, and Wally Melnitchouk of Jefferson Lab. While Jefferson Lab's beam energy is relatively low for this type of experiment, the high luminosity available has allowed many high-precision structure-function

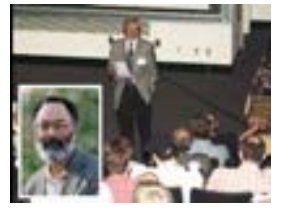

Christoph Leemann results to be produced. An interesting feature of the Jefferson Lab data is that if one scales the smooth deep-inelastic cross-section results from high-energy physics to the laboratory's kinematics, the scaled results will pass through the average of the resonant peaks of the laboratory's data. This effect, known as duality, may lead to a better understanding of how the underlying quarks and gluons link to the nucleon-meson models.

Krishna Kumar of Massachusetts and Michael Ramsey-Musolf from the California Institute of Technology presented the parity-violation experiments, where the strange quark distributions in the proton can be extracted by measuring the extremely small asymmetry in the elastic scattering of polarized electrons from an unpolarized proton target. One series of these experiments has already been completed at Jefferson Lab and several more are planned, including the G0 and HAPPEX-II experiments scheduled for next year.

Deep exclusive reactions - experiments done in deep-inelastic kinematics but where the detection of multiple particles allows the final state of the system to be determined - were presented by Michel Garcon of SPhN/Saclay and Andrei Belitsky of Maryland. Generalized parton distribution models, which should enable a complete description of the nucleon's quark and gluon distributions to be extracted from this type of data, were presented along with the results of the deeply virtual Compton scattering experiments at HERMES, DESY, and at Jefferson Lab. The results indicate that generalized parton distributions can indeed be extracted from this type of data. Several high-precision experiments are also planned for the coming years.

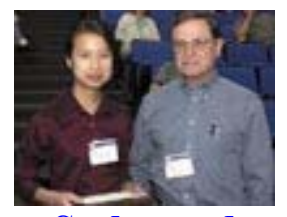

$\underline{\text { Stoler and }}$ Zheng
Steve Dytman of Pittsburgh and Simon Capstick of Florida State presented the wealth of hadron spectroscopy data that is coming from Jefferson Lab. The analysis of the vast set of data produced by the laboratory on the nucleon resonances has been only partially completed, but hints of new states are already emerging and work on a full partialwave analysis of the data is now getting underway.

Following the physics presentations, Larry Cardman, the Jefferson 
This talk focused primarily on upgrading the CEBAF to a $12 \mathrm{GeV}$ electron machine and building a fourth experimental hall. The higher energy will allow Jefferson Lab to continue its mission of mapping out the transition from the low-energy region where matter can be thought of as made of nucleons and mesons, to the high-energy region that reveals the fundamental quark and gluon nature of matter.

\section{Further reading}

Copies of these presentations can be found at www.jlab.org/div_dept/physics_division/talks/Users_meeting_2003.html.

\section{Author:}

Douglas Higinbotham, Jefferson Laboratory. 